\title{
Process Optimization Time for a Service in 4G Network by SNMP Monitoring and IAAS Cloud Computing
}

\author{
Yassine El Mahoti \\ Laboratory of Computer \\ Science, Operations Research and Applied \\ Statistics. \\ Abdelmalek Essaadi University, \\ Tétouan, Morocco \\ Souad Amjad \\ Laboratory of Computer \\ Science, Operations Research and Applied \\ Statistics. \\ Abdelmalek Essaadi University, \\ Tétouan, Morocco
}

\author{
Noura AKNIN \\ Laboratory of Computer \\ Science, Operations Research and Applied \\ Statistics. \\ Abdelmalek Essaadi University, \\ Tétouan, Morocco \\ Kamal Eddine El Kadiri \\ Laboratory of Computer \\ Science, Operations Research and Applied \\ Statistics. \\ Abdelmalek Essaadi University, \\ Tétouan, Morocco
}

\begin{abstract}
This paper discusses the energy consumption problem of $4 \mathrm{G}$ network devices that use a much higher energy compared to the $3 \mathrm{G}$ network for example, due to heavy applications that require multiple resources.To overcome this problem, a combination was proposed between network monitoring in $4 \mathrm{G}$, which is based on SNMP protocol for sorting equipment available in the network and use them in an IaaS cloud computing. This combination permits having a minimized time activity of a process and therefore reduces the energy consumed by a client for asked service.
\end{abstract}

\section{General Terms}

Cloud Computing, Monitoring, SNMP

\section{Keywords}

Cloud computing, IaaS, 4G, 3G, monitoring, SNMP, energy, Service, resources, OoS

\section{INTRODUCTION}

Last years, there has been a significant evolution in the field of information technology, driven by marketing and the emergence of communication devices (such as PDAs, laptops, the notebooks, etc.).[1]

But the increasing use of internet applications on smartphones and other devices leads to an explosion of data traffic on mobile networks.

The $4 \mathrm{G}$ network for example, ongoing research, is a future standard of telecommunication, which aims to improve the QoS, mobility, rate, etc. However, there are some goals of 4G, which are very difficult to perform, such as providing very high data rates for users moving at high speed, or guarantee a transparent handover without interruption for the users.
So, despite the enormous progress seen in recent years by this mobile telecommunication system, these efforts remain insufficient to cope with some problems.

One of these problems is the Energy Consumption, because the equipment in $4 \mathrm{G}$ network, benefit from the powerful applications and services, and therefore robust resources to handle this traffic. The goal of this article present a system able to provide a multiple services as known the $4 \mathrm{G}$ network but in the same time minimize the amount of energy consumption and uptime of a service requested by a client.The paper is organized as follows: Section 1 gives an introduction in monitoring network of $4 \mathrm{G}$ based in SNMP protocol, Section 2 gives a background and information about cloud computing: his types, his deployment methods, Section 3 gives a combination between monitoring with SNMP and Cloud Computing.

\section{NETWORK MONITORING}

Monitoring is an essential tool to monitor any network, for example to know the number of available equipment, sorting equipment according to their resources, etc. [2]

This technique become more and more crucial when the number of equipment multiplies, it allows a real-time diagnostics for equipment state for remote networks.

So why, these recent years, companies do not hesitate to invest in a product that will monitor and better manage their networks. The editors are started in the race for product supervision.

As the $4 \mathrm{G}$ network based on IP network heart, so we can monitor all devices through SNMP (Simple Network Management Protocol) SNMP is a communication protocol that allows managing network devices, monitoring and diagnosing network problems and hardware remotely.

Each device contains hardware information, configuration parameters, performance statistics like: CPU, RAM, DATA, 
etc., this information is classified as a kind of database defined by ISO1 called MIB ("Management Information Base") as you can see in the Figure 1. SNMP enables dialogue between the supervisor and the agents to collect the desired objects in the MIB. [3], [4].

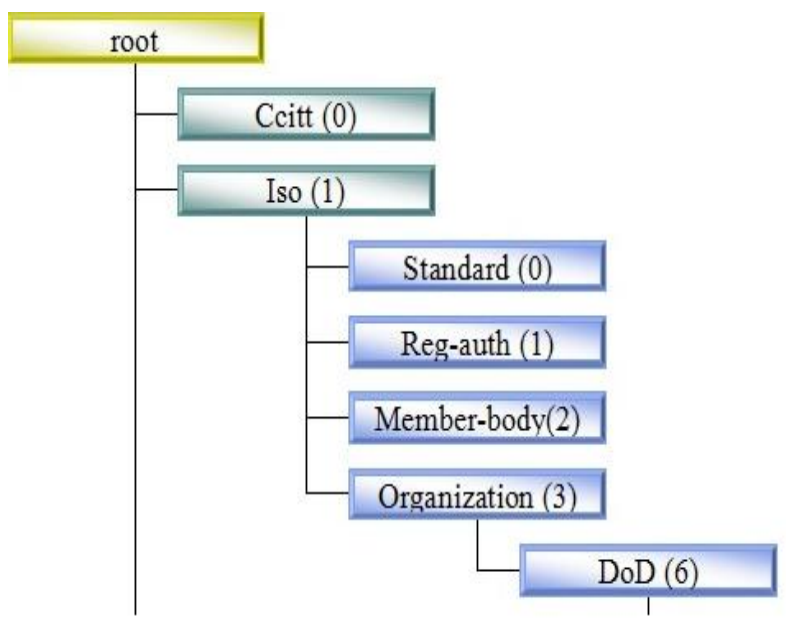

Fig 1: Management Information Base (MIB)

Each information stored in the MIB is characterized by an identifier: OID (Object Identification) OIDs are universal identifiers, represented as a sequence of integers. They are organized in hierarchical form. The IETF has proposed to represent the sequence of integers constituting OIDs separated by point.

The purpose of this article in first is collect information about network devices of $4 \mathrm{G}$ such as: RAM, CPU, hard disc occupation and sort them in a database in a way upward like in the Table 1 .

Table 1. Sorting equipment according to their use of resources.

\begin{tabular}{|l|l|l|l|}
\hline Equipment & RAM & CPU & Hard Disc \\
\hline $\mathrm{N}^{\circ} 1$ & $30 \%$ & $30 \%$ & $10 \%$ \\
\hline $\mathrm{N}^{\circ} 2$ & $30 \%$ & $30 \%$ & $10 \%$ \\
\hline $\mathrm{N}^{\circ} 3$ & $35 \%$ & $30 \%$ & $15 \%$ \\
\hline $\mathrm{N}^{\circ} 4$ & $40 \%$ & $45 \%$ & $30 \%$ \\
\hline$\ldots \ldots$ & $\ldots \ldots$ & $\ldots \ldots$ & $\ldots \ldots$ \\
\hline $\mathrm{N}^{\circ} \mathrm{n}$ & $90 \%$ & $99 \%$ & $90 \%$ \\
\hline
\end{tabular}

\section{CLOUD COMPUTING}

"Cloud computing" is a neologism used to describe the combination of the Internet ("cloud," the cloud) and the use of information technology ("computing"). It is a way to use the computer in which everything is dynamically coupled and scalable, which resources are provided as services over the Internet. Users don't need any knowledge or experience related to the technology behind the services offered. [5]

In fact, the concept of cloud computing is not new. We find the first traces in the 1960s, when John McCarty stated that computing power is available to the public in the future. The term itself is most commonly appeared around the end of the twentieth century and it seems that Amazon.com is one of the first to be assembled data centers and provides access to customers. Companies like IBM, Google and some universities have only begun to take a serious interest around 2008, when the cloud computing concept has become "fashionable".

The use of cloud differs from one company to another, from one person to another, it is essential that the client must have a solid background on the concept of cloud for benefit to this services in the right direction and not to fall into problems that may have heavy damage on the activities of the company, for examples some companies need machines to perform calculations as saying that other companies have requirements management applications, etc.

So companies or clients are required to know the type of service that will improve in their systems, and there are three types of cloud:

- SaaS: Software as a Service

- PaaS: Platform as a Service

- IaaS: Infrastructure as a Service

\subsection{SaaS: Software as a Service}

SaaS as shows the Figure 2, is when a customer has all functionalities of a program without installing it locally on his machine. SaaS is typically accessed by users using a thin client via a web browser.

The difference between SaaS and ordinary software is essential. Indeed, the SaaS offering operational software, ready to use, without passing through a stage of installation, and no maintenance task. [5], [6], [7].

Today, many businesses and organizations benefit from Saas offered by Google or Sales force for example: (Google document, Gmail, Photoshop Express), for services as customer management, human resources, project management, web conferencing, Help Desk, Wikis, blogs. Thanks to SaaS many companies uses a lot of applications without the slightest concern about the state of their computer systems, because everything is controlled by the supplier.

\section{Clients}

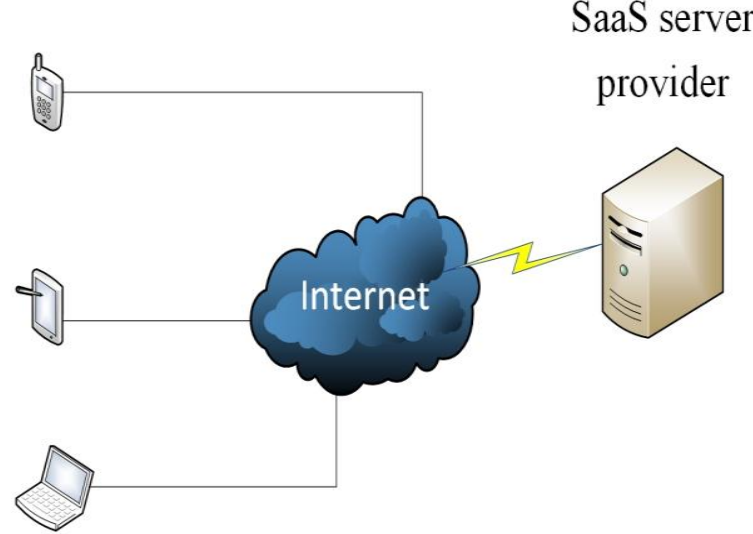

SaaS provides a piece of software from the service provider

Fig 2: SaaS, Software as a Service 


\subsection{Paas: Platform as a Service}

PaaS Platform as a Service means. This term refers to a platform run by an operator hosted and accessed via the Internet. This platform can be used to execute SaaS, and may also be available to companies who wish to host their applications from specific developments.[5], [6], [7]

PaaS services include application design, development, testing, deployment and hosting as is shown the Figure 3. PaaS providers include for example: Google App Engine [10], Microsoft Windows Azure [6] and Force.com [6].

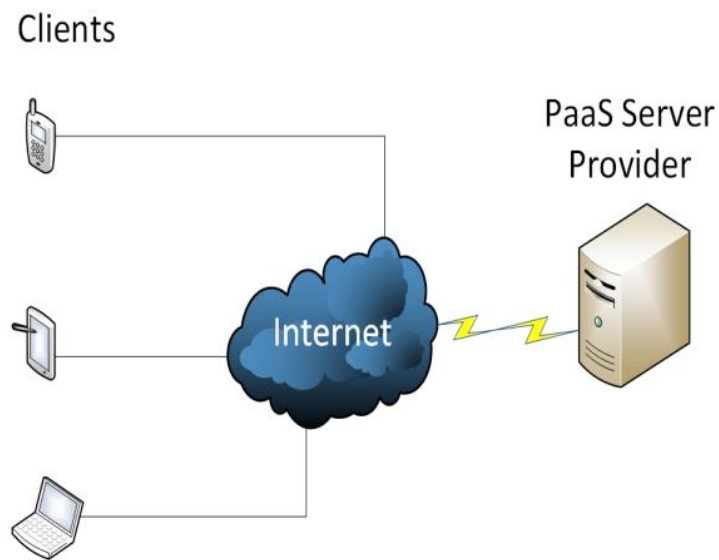

PaaS allows clients to access a computing platform over a cloud computing solution

Fig 3: PaaS, Platform as a Service

\subsection{IaaS: Infrastructure as a Service}

The term "infrastructure as a service" (IaaS) is often used to describe this form of cloud computing. This expression is derived from the first used in 2006: "Hardware as a service" (Haas). Companies buy additional infrastructure on the Web as a service. The provision of a storage or additional processing "Web" is, of course, much faster than if the supplier provides and installs it in the business. However, it requires very fast network connections. [5], [6], [7].

The following figure (Figure 5) gives some services offered by this type of cloud.

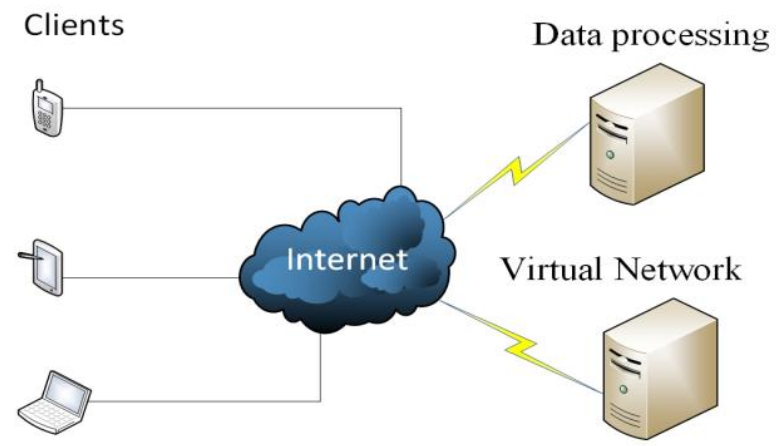

IaaS allows service provider to rent hardware resources

Fig 4: IaaS, Infrastructure as a Service
The Cloud is a topical environment that knows huge changes; diversity of services enables customers to have a fairly comprehensive list, a figure below (Figure 4) we show the different services that can be benefited by the customer.

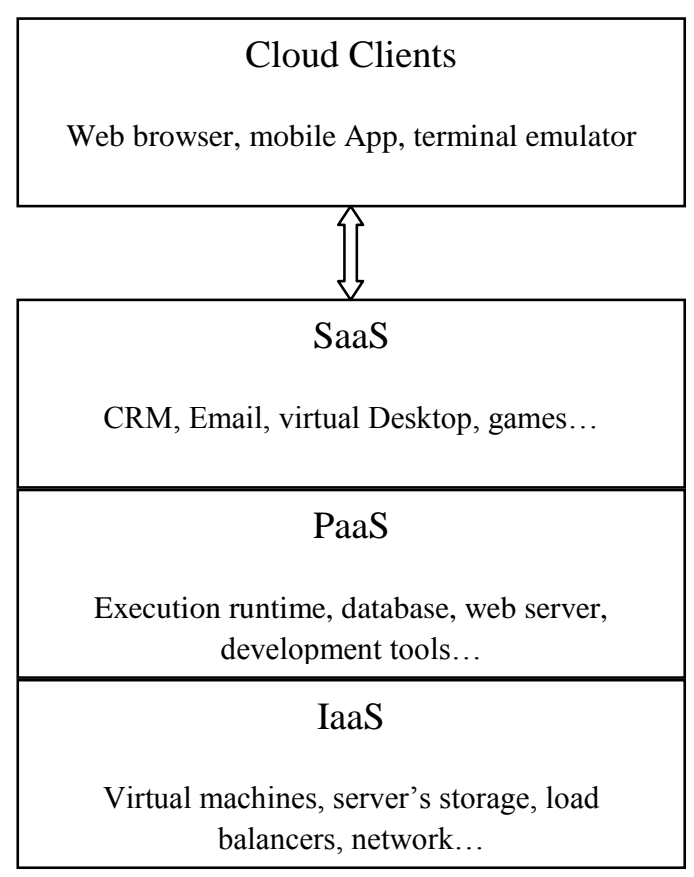

Fig 5: Cloud Computing Services

\subsection{Deployment Models}

Each company has the liberty to choose a specific deployment model for her cloud computing solution based on their specific business, operational, and technical requirements. There are three type of deployment mode: [8]

- Public Cloud

- Hybrid Cloud

- Private Cloud

\subsection{1) public cloud}

Public cloud is the cloud infrastructure which is open in internet and everybody can benefit from its services.

\subsection{2) private Cloud}

Private cloud is the cloud infrastructure which is dedicated solely to a specific customer or company.

\subsection{3) Hybrid Cloud}

Hybrid cloud is a combination of both public and private cloud models. It has the ability to strengthen a private cloud with the resources of public cloud.

NB. It is possible, as indicated in Figure 6, to combine with these three types of deployment to have a system that meet to a specific needs and requests of companies. 


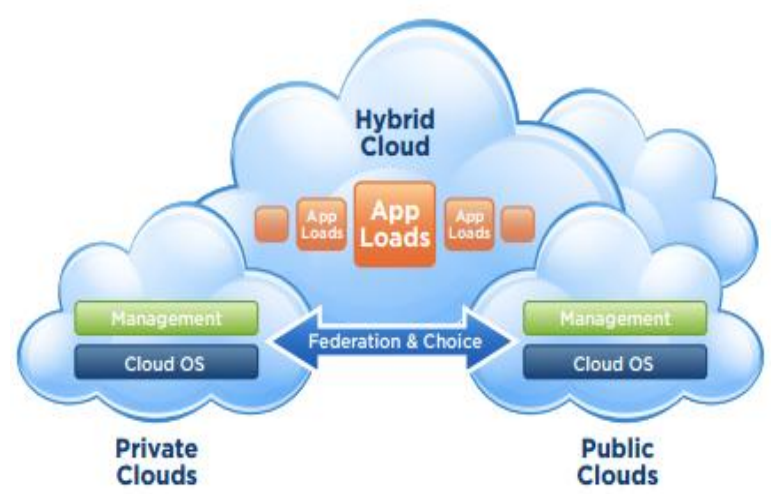

Fig 6: Cloud deployment methods

\section{ALGORHITM \& RESULTS}

To address the problem of the energy consumption for network devices $4 \mathrm{G}$, we have already pointed out that we use:

- $\quad$ SNMP monitoring to select the equipment that is in idle mode and sort them like results as in Table $\mathrm{N}^{\circ} 1$.

- Use this selected equipment the IaaS cloud computing and transform them to virtual machines to divide the service requested by the customer.

\subsection{Algorithm}

The process time witch is the time taken for a particular request asked by the client is calculated by the requested application divided by the resources equipment available in network:

$$
P_{t}=R_{e q} / R_{e s}
$$

$P_{t}:$ Process time, $R_{e q}:$ requested application asked by the client, $\boldsymbol{R}_{e s}$ : resources equipment available for executing the process.

In case there is no cloud computing:

$\mathrm{N}=1$, witch $\mathrm{N}$ is number of equipment so :

$$
P_{t}=R_{e q} / R_{e s 1}
$$

In case there is IaaS cloud computing

$\mathrm{N}=\left[11_{x} 2_{n \ldots \ldots} \ldots m\right\}$, witch $\mathrm{m}$ is the number of equipment available in the network, so the request will be divided by all equipment:

$$
P_{t}=R_{e q} / \sum_{i=1}^{m} R_{e s m}
$$

And therefore the execution time of the process deprived.

But when performing the monitoring in SNMP, you get only the equipment in idle mode and therefore the maximum resources available in the network.
So: $R_{e s}=R_{e s \max }$ (4)

Consequently, there will have a very small value of the process time $P_{t}$.

\subsection{Results}

To achieve these results, a script was developed (language C) that calculates in millisecond the process time execution in terms of number of equipment and there resources:

- $\quad$ The first results are obtained just by using cloud. monitoring.

The second are obtained by using cloud and SNMP

The next table (table2) shows the results which are obtained according to the number of equipment.

Table 2: Results of calculation process time (millisecond)

\begin{tabular}{|c|c|c|}
\hline $\begin{array}{c}\text { Equipment } \\
\text { Number }\end{array}$ & $P_{t}$ (Without SNMP) & $P_{t}$ (IAAS+SMNP) \\
\hline 1 & 5281 & 4584 \\
\hline 2 & 2995 & 2731 \\
\hline 4 & 2527 & 2153 \\
\hline 8 & 1934 & 1555 \\
\hline 10 & 1825 & 1313 \\
\hline 16 & 1716 & 1131 \\
\hline 32 & 1435 & 833 \\
\hline
\end{tabular}

As you can see in the Figure 7, the value of process time become increasingly smaller when the number of equipment is growing up, but the system knows its best performance when using only the strong equipment (equipment from the Selected SNMP).

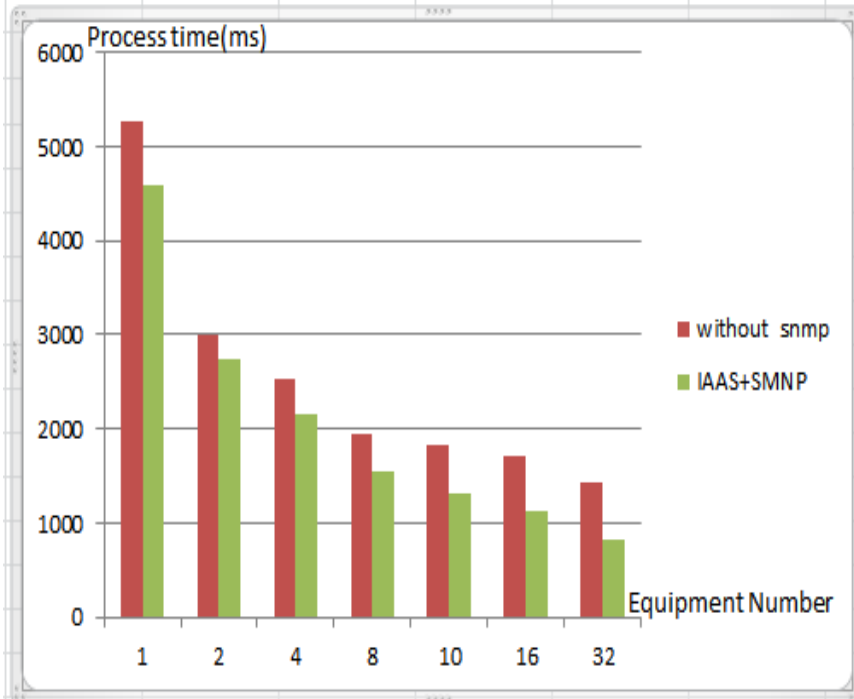

7: calculation process time by request 


\section{CONUCLUSION \& FUTURE WORK}

The cloud has become a major actor in the information technology in the word; it has changed the way of processing information by giving for these customers the liberty to choose their needs in the form of on-demand services.

Using supervision in a cloud infrastructure will get us a very reduce uptime of a process and therefore minimize the energy used and finally minimize the cost of service asked by the customer.

Based on these results, the overload of equipment is reduced; another study will be made in the future and aim to introduce another technique to minimize the overhead of Cloud server by introducing a smart load balancer to take this system to his best performance.

\section{REFERENCES}

[1] Xavier Etchevers, Thierry Coupaye, Fabienne Boyer and Noël de Palma. 2011. Auto-configuration d'applications réparties dans le nuage.

[2] David Marchett, "A Statistical Method for Profiling Network Traffic", Proceedings of the Workshop on Intrusion Detection and Network Monitoring Santa Clara, California, USA, April 9-12, 1999.

[3] Simple Network Management Protocol (SNMP), Internetworking Technology Overview, June 1999.

[4] Edmund Wong. 1997. Network Monitoring Fundamentals and Standards.

[5] RajkumarBuyya, ManzorMurshed, "GridSim: a toolkit for the modeling and simulation of distributed resource management and scheduling for grid computing", Concurrency and Computation: Practice and Experience 14, pp. 1175-1220, 2002.

[6] Mary SumiKurianand S.P.JenoLovesum. 2013. Cloud based Scaling of Grid Resources through Grid Middleware.
[7] Tan, Z., Gurd, J. R.: Market-based Grid resource allocation using a stable continuous double auction. In: 8th International Conference on Grid Computing. IEEE Computer Society Press, Austin, Texas, USA (2007).

[8] Buyya, R. , Abramson, D. , Venugopal, S. (2005) "The Grid economy" Proc. I. E. E. E. 698-714 .

[9] Nicolas Grevet. 2009. Le cloudcomputing: évolution ou révolution? Pourquoi, quand, comment et surtout faut-il prendre le risque?

[10] Ajith Singh. N, M. Hemalatha. 2012. An Approach on Semi-Distributed Load Balancing Algorithm for Cloud Computing System.

[11] Eddy Caron, FrédéricDesprez, "DIET: a scalable toolbox to build network enabled servers on the grid, High Performance Computing Applications", International Journal of High Performance Computing Applications, vol. 20, issue. 3, pp. 335-352, 2006.

[12] Erwan Daubert, Françoise André, Olivier Barais. 2011. Adaptation multi-niveaux: l'infrastructure au service des applications.

[13] Daniel Nurmi, Rich Wolski, Chris Grzegorczyk, GrazianoObertelli, SunilSoman, Lamia Youseff, DmitriiZagorodnov, "The Eucalyptus open-source cloudcomputing system", 9th IEEE/ACM International Symposium on Cluster Computing and the Grid, CCGRID’09,pp. 124-131, 2009.

[14] François Tonic. 2009. Stratégie et révolution de l'infrastructure informatique, de la manière de concevoir les applications et leur consommation dans le nuage sous forme de services.

[15] SandeepTayal, "Tasks Scheduling optimization for the Cloud Computing Systems," International journal of No.5, Issue no. 2, pp 111 - 115, 2011. 